\title{
Study of Phase Circle Map Model and its Symbolic Dynamics
}

\author{
Z. Chen \\ Department of Automation \\ Zhejiang University City College \\ Hangzhou, Zhejiang, P.R China
}

\author{
S.W. Pan \\ Department of Automation \\ Zhejiang University City College \\ Hangzhou, Zhejiang, P.R China
}

\begin{abstract}
Integrate-and-Fire Mode (IFM) is a quite simple neuron model, however, it is possible to simulate circle map. With the aid of IFM, the circle map iteration of phase can be implemented, meanwhile the symbolic sequences are acquired conveniently from Integrate-and-Fire process. In this paper, the study of phase circle map model and its symbolic dynamics is made. Firstly, the partition of symbols and their acquiring way are introduced one after the other. And then, the rotation and the devil staircase are computed immediately to distinguish the working states from the symbolic sequences. Finally, a distance formula of symbolic sequence, by which the relationship between the initial phase and distance can be shown, is given. It is shown that above analysis provides a simple and convenient way for the application of circle map.
\end{abstract}

Keywords- neuron model; circle map; integrate-and-fire model; symbolic dynamics

\section{INTRODUCTION}

Circle map is the mapping of a circle to itself. Comparing with single parameter maps such as Bernoulli map and sawtooth map, circle map has two parameters which exhibits richer dynamic characteristics, e.g., the periodic state, frequency locking and so on [1]. Single parameter maps have been widely used in signals measurement and processing. Since they are all amplitude mapping, and processing are for amplitude $[2,3]$. However, for many time-domain signals, frequency and phase also contain useful information. The most typical example is the biological information system where the pulse signal is embedded with information in interval.

When measuring and processing signals with nonlinear maps, the more important thing is that the system should be very simple and felicity, such as down saw-tooth map [4], which is implemented by only charging and discharging to a capacitor. If the iteration of a map is imitated by complex calculation units, such a model is applicable only for simulation studies and not practical at all. Integrate-and-Fire Mode (IFM), which was proposed as a simple model of pulse neuron by Keeper and others [5, 6], exports a spike train. In order to describe this sort of signals effectively, the symbolic dynamics theory is used. The spike trains are expressed with symbolic sequences, which are digital data and thus may be used to calculate parameters of spike trains $[7,8]$.

In this paper, the IFM-based phase circle map and its symbolic dynamics are first described, and then the obtained symbol sequences are used to study the system. With the aid of symbolic sequences, the working states of the system, such as period, quasi-period and chaotic state, may be determined, and the rotation and the devil stairs can also be computed. Based on symbolic sequence order rules of circle map, a distance formula of symbolic sequence is defined.

\section{OUTLINE OF CIRCLE MAP [9]}

Circle map is a simple mathematical model which describes relaxation oscillation driven by periodic external force whose iterative equation is

$$
\left\{\begin{array}{l}
\theta_{n+1}=f_{\Omega, K}\left(\theta_{n}\right)=\theta_{n}+\Omega+g\left(\theta_{n}, K\right) \\
g\left(\theta_{n}, K\right)=g\left(\theta_{n}+1, K\right)(\bmod 1)
\end{array}\right.
$$

in which $\Omega$ is the period ratio of the extern excitation to intrinsic oscillation, $K$ represents coupling strength of external excitation and the system, and $g()$ is a periodic function associated with the excitation signals. When $g()$ is a sinusoid function, eqn(1) is transformed into a standard circle map, or sine-circle map, as follows:

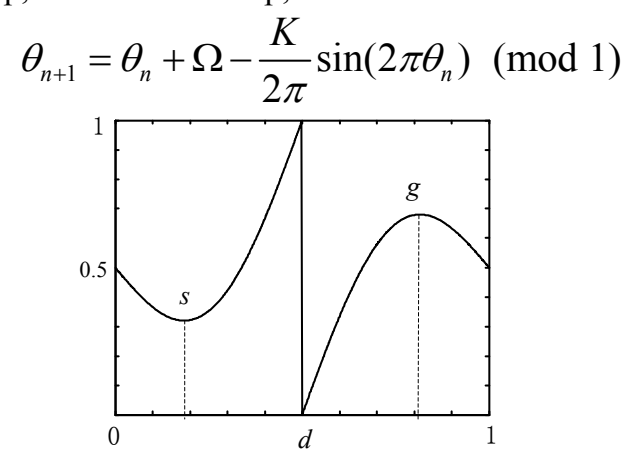

FIGURE I. STANDARD CIRCLE MAP.

Fig.1 demonstrates a standard circle map. Comparing with single parameter maps, the circle map has more complex dynamical behavior, such as periodic, quasiperiodic and chaotic motions, etc. Usually, an Arnold tongue in $\Omega-K$ plane is used to vividly express frequency-locking area and rotations. When $K<1$, the map is monotonic, otherwise the map becomes non-monotonic, and there may be chaos existed. 


\section{ITERATIVE RELATIONSHIP OF IFM}

To better understand the problem, a normalized IFM for which the main working principle is shown in fig.2, is considered. The state of IFM represented by $x(\tau)$ corresponds to the membrane potential of a neuron [5]. $x(\tau)$, which is usually a capacitor voltage, is charged up by a constant current source $\mathrm{s}_{0}$. The base threshold $b(\tau)$ is always less than the unvarying firing threshold. It is implied that the state instant reduces to the base threshold when it reaches the firing threshold, and simultaneously output a pulse. Repeated in this manner, the IFM outputs a spike train $y(\tau)$. When the base threshold is hold constant, the IFM simply repeats a limit cycle of slow charging and instantaneous discharging indefinitely. A simple but significant situation is to introduce a sinusoidal oscillation into the base threshold, i.e., $b(\tau)=$ $k_{b} \sin (2 \pi \tau)$ where $k_{b}$ is the amplitude of the sinusoidal oscillation, as shown in fig. 2 .

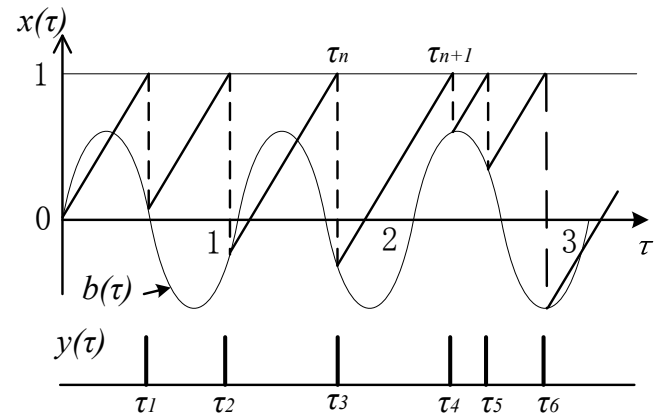

FIGURE II. THE WORKING PRINCIPLE OF IFM.

The process can be described by the following eqns(3)\&(4)

$$
\begin{aligned}
& \begin{cases}\frac{d x}{d \tau}=s_{0} & \text { for } x(\tau)<1 \\
x\left(\tau_{+}\right)=b\left(\tau_{+}\right) & \text {if } x(\tau)=1\end{cases} \\
& y\left(\tau_{+}\right)= \begin{cases}0 & \text { for } x(\tau)<1 \\
1 & \text { if } x(\tau)=1\end{cases}
\end{aligned}
$$

Where $x$ is the state of the IFM, $b(\tau)$ is the base threshold whose amplitude satisfies $0<k_{b}<1$, and $s_{0}$ is a positive constant that represents the constant buildup rate of the state $x$.

Consider the dynamics of the spike train and integrate eqn (3) on $\left[\tau_{n}, \tau_{n+1}\right]$, one obtains the following recursion:

$$
\tau_{n+1}=\tau_{n}+\frac{1}{s_{0}}\left(1-b\left(\tau_{n}\right)\right)
$$

Replace the base threshold with a sinusoid signal, and let $\Omega=1 / s_{0}, K=2 \pi k_{\mathrm{b}} / s_{0}$, one obtains

$$
\tau_{n+1}=\tau_{n}+\Omega-\frac{K}{2 \pi} \sin \left(2 \pi \tau_{n}\right)
$$

Let $\theta_{\mathrm{n}} \equiv \tau_{\mathrm{n}}(\bmod 1)$, one obtains the standard sine-circle map which has the same expression as eqn(2). In above equation, $\theta_{n}$ is referred to as the normalized phase, and the parameter $\Omega$ is the rotating frequency of $\theta_{n}$ in the absence of the nonlinear term, which can be controlled by the buildup rate $\mathrm{s}_{0}$. The parameter $K$ determines the strength of the nonlinear term and can be controlled by the amplitude of the base threshold when $\Omega$ is fixed. In conclusion, the IFM can be used to compute the standard circle map for any combination of $\Omega$ and $K$.

\section{SyMBOLIC DYNAMICS OF THE IFM}

\section{A. Partition the Symbols}

Although symbolic sequence is a coarse-grained description of dynamics, it provides the more practical and efficient way to study nonlinear dynamical systems $[10,11]$.

The key of the works in this way is the acquisition of symbolic sequences. Fig. 3 demonstrates a schematic diagram how to partition the symbols of circle map and IFM. According to the symbolic dynamic theory [9], the function of circle map has three special points, minimal point $s$, maximal point $g$ and a point of discontinuity $d$ which divide the interval $[0,1]$ into four branches. We assign symbols 0,1 , 2,3 in turn for each branch where symbols 1 and 2 correspond to increasing branches and are stipulated as even while symbol 0 and 3 correspond to decreasing branches and are odd. When $K<1, s \rightarrow 0, g \rightarrow 1$, the symbols degenerate into 1 and 2. For a phase sequence $\Theta=\left\{\theta_{0}, \theta_{1}, \theta_{2}, \ldots, \theta_{n}, \ldots\right\}$, there is a corresponding symbolic sequence $S=\left\{s_{0}, s_{1}, s_{2}, \ldots\right.$, $\left.s_{n}, \ldots\right\}$, where $s_{i} \in\{0,1,2,3\}$ is determined by the branch in which $\theta_{i}$ locates. In practice, instead of directly measuring the phase $\theta_{i}$, one obtains the symbol $s_{i}$ by analyzing the logical relation of $x(\tau)$ and $b(\tau)$ when the IFM is in the firing process.

Taking the derivative of eqn(2) with respect to $\theta_{n}$, and setting the result to zero, one obtains

$$
\frac{d \theta_{n+1}}{d \theta_{n}}=1-K \cos \left(2 \pi \theta_{n}\right)=0
$$

For $d \theta_{n+1} / d \theta_{n}=0, \theta_{n}$ is at extreme points $s$ and $g$ of circle map, while for $1-K \cos \left(2 \pi \theta_{n}\right)=0$, the solutions represent that the derivative of the base threshold equals to the slop $s_{0}$, marked as $s$ and $g$ in Fig.3. The first equation of eqn(7) indicates that these points are coincident, i.e., $s=s^{\prime}, g=g^{\prime}$. Thus, we may determine the range of phase $\theta_{n}$ by making logical judgment to IFM.

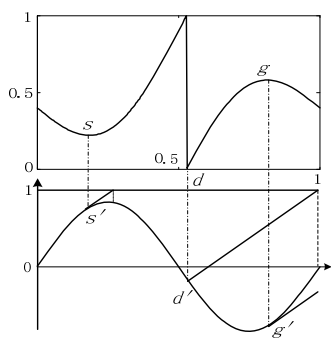

FIGURE III. IFM CORRESPONDING TO CIRCLE MAP. 
Within the ranges [0,s) and (g,1], $d \theta_{n+1} / d \theta_{n}<0$, and the gradient of $b(\tau)$ is greater than the slop $s_{0}$. This can be done using a comparator to judge the states immediately following the firing of IFM. If $b(\tau+d \tau)>x(\tau+d \tau)$, at the same time $b(\tau)$ is in its positive half cycle, then the phase point locates in $[0, s)$, and the symbol is 0 ; otherwise the phase point locates in $(\mathrm{g}, 1]$, and the symbol is 3 . While in the ranges $[\mathrm{s}, \mathrm{d})$ and $[\mathrm{d}$, $\mathrm{g}]$, the case is just the opposite. Furthermore, if the successive firing point is in the same period of $b(\tau)$ as former firing, the phase point locates in [s, d), and the symbol is 1 ; otherwise if the successive firing point is in the next period of $b(\tau)$, the phase point locates in [d, g] and the symbol is 2 . Therefore, the symbolic sequence $S=\left\{s_{0}, s_{1}, s_{2}, \ldots, s_{n}, \ldots\right\}$ can be obtained by differentiating the states after firing of IFM, and there is no need to measure phase sequence $\Theta=\left\{\theta_{0}, \theta_{1}\right.$, $\left.\theta_{2}, \cdots, \theta_{n}, \cdots\right\}$.

If $K<1$, there is none of $b\left(\tau_{+}\right)>x$ state, and only symbols 1 and 2 may be acquired. When $K>1$, there are symbols 3 or 4, and this time the circle map may be turned into chaos. As a result, it can also be done to estimate if the system may be chaotic from the sorts of symbols.

\section{B. Calculation of Rotations}

An important character of circle map is rotation. The definition of the rotation for the $\operatorname{map} \tau_{n+1}=f\left(\tau_{n}\right)$ is given by

$$
\rho=\lim _{n \rightarrow \infty} \frac{1}{n}\left[f^{n}\left(\tau_{0}\right)-\tau_{0}\right]
$$

What is reflected in by the rotation $\rho$ is the average increment of once iteration, with which it can be done to mainly estimate the behavior of the circle map under certain parameters and working conditions. In some sense it has the same function as Lyapunov exponent. However, it may be easier to obtain the rotation using symbolic sequence[10]. Each time when the symbols 2 or 3 are appeared in the sequence, it indicates that a period has turned over. Therefore, the rotation can be calculated by

$$
\rho=\lim _{n \rightarrow \infty} \frac{N_{2}+N_{3}}{N}
$$

Where $N$ is the total number of symbols, $N_{2}$ and $N_{3}$ are the counts of symbols 2 and 3 , respectively. The rotation of a period state may be expressed as a rational number $p / q$, which is easier to be obtained by eqn(9). The minimum symbols number of duplicate subsets in a symbolic sequence is its period $q$, the sum of symbols 2 and 3 in a period equals $p$. and then $\rho=p / q$. When $k \leq 1$, there are only two symbols 1 and 2, the system can be in periodic or quasi-periodic state. On the other hand, when $k>1$, there may be 3 or 4 symbols, and the system could be in periodic or chaotic states. So it is easier to distinguish the working states of IFM with symbolic sequence.

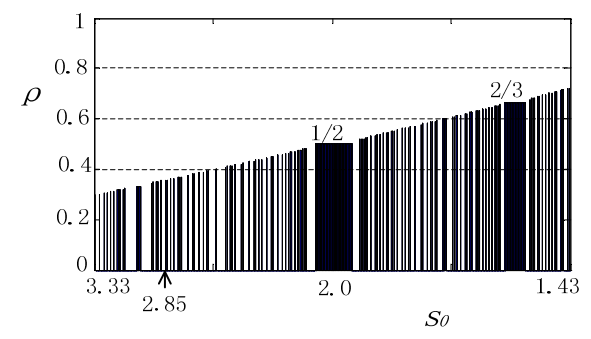

FIGURE IV. ROTATION DISTRIBUTION.

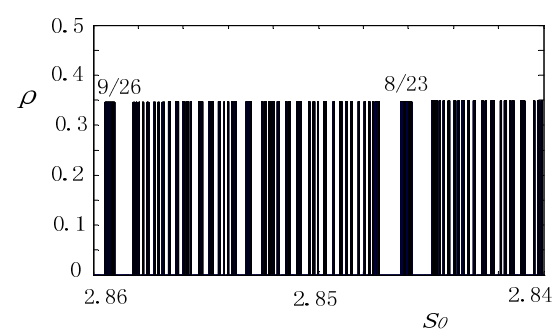

FIGURE V. LOCAL DISTRIBUTION OF ROTATION.

Fig.4 shows the variation of rotation with $s_{0}$ varying among [3.33, 1.43], which is computed by eqn(9) and quite resemble devil stair of circle map. For clarity, in fig.4 only rotations whose period less than 50 are drawn. Finer structure is shown in fig.5 where the range [2.86, 2.84] is extended, and periods are increased to 100 . Theoretically, the periods may be infinity.

\section{The Order and the Distance of Symbols}

There is a correlative relationship between the symbolic sequences and the output signals of the system. Although signals are different in varying degrees, they have not orders, or they cannot compare the sizes with each other. According to the symbolic dynamical theory, for a dynamic system to which the equation is known, its symbolic sequences can not only compare the sizes with each other using certain rules, but define the distance between the sequences [12] as well.

For the circle map, the order of a single symbol is the same as symbol values that is $0<1<2<3$. The symbolic sequence is composed of many symbols, the order rule is deduced by the increasing and decreasing branches of the circle map [9]:

If the longest common word of two symbolic sequences is even, the order of the sequence is according to the first different symbol; otherwise the order is in reverse with the first different symbol.

Based on this rule, we propose a definition of the distance from symbolic sequence $S_{a}=\left\{a_{\mathrm{i}}\right\}$ to $S_{b}=\left\{b_{\mathrm{i}}\right\}$, as follows:

$$
d\left(S_{a}, S_{b}\right)=\sum_{i=1}^{\infty}\left[\left(a_{i}-\frac{3}{2}\right)(-1)^{A_{i-1}}-\left(b_{i}-\frac{3}{2}\right)(-1)^{B_{i-1}}\right] / 4^{i}
$$

Where $A_{i-1}$ and $B_{i-1}$ denote the parity of the first $i-1$ symbols of sequences $S_{\mathrm{a}}$ and $S_{\mathrm{b}}$ respectively. By this definition, for the circle map, it can be verified that when the 
maximal sequence is $S_{\max }=\{303030 \ldots\}$ and the minimal sequence is $S_{\min }=\{030303 \ldots\}$, then the distance of them is 1 .

\section{The Relation of Initial Phase and Distance}

The phenomenon that sensitivity to initial conditions leads to chaotic system is well-known [13], and have been widely used in signals detection $[2,4]$. So far these initial sensitivity based detections are all for amplitude maps, and the signals processed are also for amplitude. Frequently, however, for many time-domain signals, frequency and phase also contain valuable information. What the IFM is implemented for is a phase circle map. By increasing the amplitude of the base threshold to $K>1$, the map becomes non-monotonic, thus there may be chaotic states. Chaotic circle map is much sensitive to the initial phase, based on this character, phase difference may be measured. The main principle of the phase measuring is similar to the one of amplitude. When the base threshold signal has a weak shift from referential signal, the output spike train of IFM will have a greater change, and corresponding symbol sequence will be more different, while the latest situation can be measured by distances.

A more direct approach is the utilization of the distance formula in eqn(10), in which the deviation of initial phase is expressed by the distance. For example, if the symbolic sequence of a zero phase signal acts as reference, then the distance between a symbolic sequence of a delayed signal and the reference is used for the measurement of the phase difference. Next the relation between initial phase of the base threshold and the distance of symbolic sequences is revealed by computer simulation of the IFM. The parameters of IFM for the simulation experiment are $s_{0}=2, k_{b}=0.7$, accordingly one obtains $\Omega=0.5, K=2.2$, showing that the system is chaotic and the output is sensitive to initial phase. Let the initial phase equal to 0 , one obtains the reference symbolic sequence $s y m b 0=01320132013201320$. Then, from 0 to $180^{\circ}$ with an interval of $0.001^{\circ}$, the symbolic sequences are acquired, and the distances to reference symb0 is calculated. Fig. 6 presents the relations of initial phase and the distance in different scales, from which the following conclusions can be drawn: (i) the distance increases monotonically with respect to the phase difference; (ii) the linear relationship is not satisfied; (iii) there are numerous irregular steps; and (iv) within different phase ranges, there is obvious self-similarity.
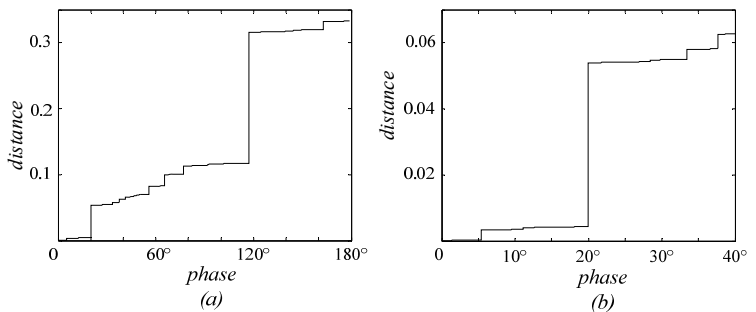
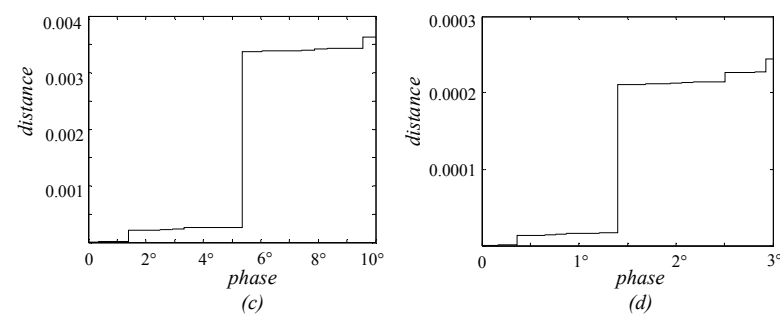

FIGURE VI: RELATIONS OF INITIAL PHASE AND DISTANCE.

\section{CONCLUSION}

In this paper, a simple model of phase circle map is constructed in time domain based on the Integrate-and-Fire Mode (IFM), and then several ways to study the circle map by using symbolic sequences acquired are presented. It can be observed from the preliminary results of the study that the method is simple and reliable. The method to determine the symbols according to the states makes the result not only precise but immune to noise as well, since there is no need to capture the spike train signals. Moreover, the symbolic sequences are digital data, and may be processed by computers easily. Consequently, the simple model as well as the symbolic tool provides a new and efficient way for theoretic and applied researches of the circle maps.

\section{ACKNOWLEDGMENT}

This work is partially supported by Zhejiang Provincial Natural Science Foundation (Grant No.Y1100119) and the Pattern Recognition Key Disciplines of Zhejiang University City College (ZUCC).

\section{REFERENCES}

[1] Chen Shigang, Circle maps, Shanghai Scientific and Technological Education Publishing House, pp.1-23, 1998.

[2] Zhao Wenli, Xia Wei, Liu Peng \& Wang Linze, Research on weak signal amplification principle and method based on chaos theory. Acta Physica Sinica, 59(5), pp.2962-2970, 2010.

[3] Zhang Lin, Wang Lianfen, Ning Xiaolei \& Chen Minghi, Research and Simulation on Weak Sine Signal Detection by Using Duffing Oscillator. Chinese Journal of Sensors and Actuators, 22(8), pp.11371141,2009

[4] Wu Wei, Fan Yingle \& Pang Quan, Study of Chaotic Measurement Based on Noise Reduction by M ulti-Coupling. Chinese Journal of Sensors and Actuators, 20(3), pp. 592-595, 2007.

[5] J.P.Keener, F.C.Hoppensteadt \& J.Rinzel, Integrate-and -fire models of nerve membrane response to oscillatory input, SIAM J. Appl. Math., 41, pp.503-517, 1981.

[6] Humberto Carrillo, Frank Hoppensteadt, Unfolding an electronic integrate-and-fire circuit. Biological Cyber- netics, 102, pp.1-8, 2010.

[7] Chen Chong, Zhang Hong \& Chen Zhuo, A study of an integrate-anddischarge model for parameter measurement with applied symbolic dynamics. Nonlinear Dynamics of Electronic Systems, Proceedings of NDES:1-4, 2012

[8] Chen Chong, Ding Jiong, Zhang Hong etc., Study of an integrate-anddischarge model with symbolic dynamics. Acta Physica Sinica,62(14), pp.140502, 2013

[9] Zhang Zhongjian \& Chen Shigang, Symbolic Dynamics of the circle map. Acta Physica Sinica, 38(5), pp.1-8, 1989.

[10] Zheng Weimou \& Hao Bailin, Applied symbolic dynamics, in Experimental Study and Characterization of Chaos vol. 3. Singapore: World Scientific, pp.110-134,1990. 
[11] Annick Lesne, Jean-Luc Blanc \& Laurent Pezard, Entropy estimation of very short symbolic sequences. Physical Review E79, pp. 046208, 2009.

[12] Wang Liming \& Dan Schonfeld, Mapping Equivalence for Symbolic Sequences: Theory and Applications. IEEE Transactions on Signal Processing, 57(12), pp.4895-4905, 2009.

[13] Ray Brown \& Chua Leon, Is sensitive dependence on initial conditions nature's sensory device. International Journal of Bifurcation and Chaos, 2, pp.193-199, 1992. 